\title{
Strengths and Weaknesses of Cognitive Theory
}

\author{
Mana Alahmad \\ Faculty of Foreign Languages, Tehran North Branch, Islamic Azad University, Tehran, Iran \\ mana.alahmad60@gmail.com
}

\begin{abstract}
This paper focuses on strengths and weaknesses of the Cognitive Theory. Many theories have been proposed over the years to explain the developmental changes that people undergo over the course of their lives. These theories differ in the conceptions of human nature they adopt and in what they regard to be the basic causes and mechanisms of human motivation and behavior. Piaget's theory of cognitive development explains how a child constructs a mental model of the world. He disagreed with the idea that intelligence was a fixed trait, and regarded cognitive development as a process which occurs due to biological maturation and interaction with the environment. Cognitive learning theory is best used to guide learning when trying to build usefulness Knowledge structures, and clinical reasoning teaching. The theory suggests that Clinical exercise is crucial to the organization of Memory Knowledge and Recall Facilitation. This means teachers will be expected to be aware of prior clinical experiences of the learner before assessing the learner's experience Powerful performance.
\end{abstract}

Keywords

cognitive development; child development; Jean Plaget

\section{Introduction}

In the last century, Jean Piaget proposed one of the most famous theories regarding cognitive development in children. Although Piaget's theories have had a great impact on developmental psychology, his notions have not been fully accepted without critique. Piaget also neglected cultural and social interaction factors in the development of children's cognition and thinking ability. Moreover, in terms of the methodological approach, Piaget's theory had some ethical and bias problems as he studied his own children.

Cognition is defined simply as a process of our thinking. It defines the very act of acquiring information through observation, thinking, imagination, memory, and judgment, problem-solving and selective attention. One of the higher functions our brain carries out is cognition. The study of perception has been a scientific subdiscipline known as cognitive psychology in recent years. Piaget (1936) was the first psychologist to make a systematic study of cognitive development. His contributions include a stage theory of child cognitive development, detailed observational studies of cognition in children, and a series of simple but ingenious tests to reveal different cognitive abilities. What Piaget wanted to do was not to measure how well children could count, spell or solve problems as a way of grading their I.Q. What he was more interested in was the way in which fundamental concepts like the very idea of number, time, quantity, causality, and justice and so on emerged. Before Piaget's work, the common assumption in psychology was that children are merely less competent thinkers than adults. Piaget showed that young children think in strikingly different ways compared to adults. According to Piaget, children are born with a very basic mental structure (genetically inherited and evolved) on which all subsequent learning and knowledge are based. Piaget's Theory differs from others in several ways: It is concerned 
with children, rather than all learners. It focuses on development, rather than learning per se, so it does not address learning of information or specific behaviors. It proposes discrete stages of development, marked by qualitative differences, rather than a gradual increase in number and complexity of behaviors, concepts, ideas, etc. The goal of the theory is to explain the mechanisms and processes by which the infant, and then the child, develops into an individual who can reason and think using hypotheses. To Piaget, cognitive development was a progressive reorganization of mental processes as a result of biological maturation and environmental experience. Children construct an understanding of the world around them, then experience discrepancies between what they already know and what they discover in their environment.

Piaget suggested that all children journey through the sensorimotor, preoperational, concrete operations, and formal operations stages of development (Moreno, 2010). During the process of moving through one stage to the next, children's cognitive ability change qualitatively (Sigelman \& Rider, 2012). Piaget also believed that cognitive development is a continuous process and all children, even in different environmental context and culture diversity around the world, have the same sequence of cognitive development (Hockenbury \& Hockenbury, 2011).

Up to the present time, the bulk of the research into the effects of second language instruction have recorded the linguistic progress of one group exposed to an FonF approach and another exposed to FonFs instruction (Ellis, Basturkmen, \& Loewen, 2002; Loewen, 2003). The present study aims to further explore this area, concentrating on the effect that FonF, and FonM have on the acquisition of a grammatical form with communicative value: the six forms of conditional sentences have been selected. Studies on the effectiveness of focus-on-form instruction have increased greatly and suddenly in recent years. Ellis et al (2002) improved Long's (1991) definition of FonF instruction by making the difference between two types: 'Planned focus-on-form' and 'incidental focus-on-form.' In many studies, focus on form was the result of prior planning on. (Alahmad, 44)

Assessment of student is one of the most important responsibilities of teachers, because the quality of teaching in classroom is closely associated with the quality of the applied assessment. Hence it is essential for teachers to possess assessment literacy. Assessment literacy is important because it helps teachers to perceive, analyze and use data on student performance to improve teaching. Stakeholders are influenced by language tests, therefore; being assessment literate is more vital for them, because assessment illiteracy results in inaccurate assessment and consequently the purposes of assessment could not be fulfilled. (Rahimirad, 10)

\section{Review of Literatures}

\subsection{Difference between Cognitive Theory and Structural Theory}

Cognitive theories are of great importance to knowledge resources and strategies for learning (attention, understanding, memory, reception, processing, and information processing). The learner's understanding of the information gained, and the way he obtained it, enhances his mathematical performance. This behavior, an individual's experience or training affects his behavior. By the following features, cognitive theories are concerned with cognitive structure: distinction, organization, coherence, integration, quantity, consistency, and relative stability. In recent years, structural theory has gained popularity though its concept is not new. 


\section{a. Piaget vs. Vygotsky}

Lev Vygotsky developed his theory on child development at the same time Piaget was developing his own theory. Like Piaget, Vygotsky believed that children develop through stages. Unlike Piaget, Vygotsky believed that learning and development were tied to social interactions and culture. Whereas Piaget believed that children learn through doing, Vygotsky believed that they learn through being shown.

\section{b. Cognitivism and Behaviourism}

Cognitivism is reflected in the work of theorists such as Dewey (1938), Piaget (1964), Vygotsky (1978), and Gagné (1970); there are, however, no clear lines separating the different theories of learning and they often blur into one another. Gagné (1968) has also been associated with behaviourism, which identifies behaviours that can be observed. The difference with behaviourism is that the entire thought processes that occur in the mind of the learner are not fully considered. The primary concern is that of stimuli and responses to those stimuli, as can be seen in the works of Pavlov (1927), Watson (1928), Thorndike (1913), Skinner (1938), and Gagné (1970). There are three types of behaviourism; methodological, psychological and analytical.

\section{c. Connectivism Theory}

Connectionism is promising to provide a better understanding of the cognitive behaviour than the classical theory because of its association to neuro-science and 'how' and 'how much' change will be brought about has been a matter of great debate in the neuroscientific circles (Garson 1997). The experiments on the connectionist models of cognitive science have demonstrated ability to pick-up things and learn skills such as reading, structure of simple grammar in language, and face recognition (Garson 1997). These experiments have been possible due to the close proximity of the models to neurology, unlike the language of thought, which concentrates of theoretical interpretation of ideas. The model uses a large number of units representing neurons connected together by weights representing the synapses to connect the neurons together. The weights can successfully link one unit to another like neurons. This significant similarity to the actual brain gives cognitive models an edge over other traditional theories. The use of connectionist models over the classic theory has many relative advantages and deals with issues like stability, sequence problems and sensitivity (Feldman 1982). The connectionist models have a lot of potential for the research into the cognitive panorama but there are certain drawbacks, which can be debated in relevance of the classical theory. The systematicity debate over the LOT and the Connectionist models of cognitive science has been around for more than a decade now. With the evolution of this debate, Fodor and Pylushyn's challenge has become the benchmark for the two theories/models (Garson 1997). And, it has come as a surprise that no one has been able to meet the challenge of providing neural network, which is capable of learning complex processing that can be further generalized to a full range of novel inputs (Garson 1997).

\section{d. Input Processing Theory}

With no doubts, Second Language Acquisition is a complex process. One of the processes which involves in SLA is IP, the initial process by which learners connect grammatical forms with their meanings as well as how they interpret the roles of nouns in relationship to verbs. IP theory captures a series of internal strategies which learners might use in comprehending sentences and how these strategies might affect acquisition. Within the last few years, investigations have been made on the effects of one type of explicit grammar instruction which focuses on the learners' processing strategies followed by input-based 
practice. In the first process, learners drive intake from input. In the second process, PI assists the learners in making form-meaning connections and it aims to improve the qualities of the input to increase the amount of input that becomes intake. (VanPatten \& Cadierno, 5660) According to Sanz and VanPatten (1998), IP refers to "a research domain about how learners make form-meaning connections as well as parse incoming sentences in the L2....It is the application of psycholinguistic inquiry to comprehension and processing of second language sentences" (p. 50). IP attends to explain the way learners get form from input and the way they assign grammatical roles to nouns during comprehension while their primary attention is on meaning.

\section{e. Behaviorism Theory}

Many critics argue that behaviorism is a one-dimensional approach to understanding human behavior and that behavioral theories do not account for free will and internal influences such as moods, thoughts and feelings. As my professor told us, behaviourism tends to be «superficial» or shallow in explaining behavior and learning. As can be inferred from the theory presented, much of the human behavior and learning abilities were attributed to the effects of external factors that serve as reinforcers or punishers. It only says that regardless of what behavior an individual learned in the past through the system of reinforcement, he can still be able to modify and/or change it when new circumstances offer new information. There are some drawbacks toward this theory as follow: It ignores the mental processes that are involved in learning unlike the cognitive approach who views these processes as important. According to the behaviourist approach, people can only learn as a result of their experiences. The use of animals in applying the laws of learning to humans has been criticized as humans are more complex than animals. The principle of operant and classical conditioning do not account for spontaneous behavior in humans. It rejects the possible role of biological factors in human behaviour, unlike the biological approach which considers nature and important factor. Behaviourists view humans has passive learners, unlike humanistic psychologists who view humans as active agents able to control and determine their own development.( Reynaldo Flores)

\section{f. Sociocultural Theory}

Vygotsky's sociocultural theory of development suggests psychological tools and semiotic signs that individuals use to mediate development reflect their social origins and cultural identities. One could argue, for example, that sign systems used to structure relationships psychological mechanisms (e.g., how one composes music or how one member touches another) reflect specific and unique cultural values and ethos of a society, community, or a group of individuals. For many indigenous groups and communities, oral and dance presentations, and/or traditional storytelling, rather than formal symbolic representations (e.g., textbooks) may take precedence in learning and development. (Phan, 2008b). Vygotsky believes that external influences cognitively transform individuals' interpretation, perceptions, and meaning of the external world. There are a number of theoretical models, for example, which situate individuals' learning and development in a holistic system made up of different internal and social factors. We contend that the interrelations between the three facets are intertwined and, more importantly, any form of development requires an input of one and the output of another. In the last couple of years, there has been an emerging trend in social sciences research with the study of amalgamation of both extraneous factors and internal cognitive, affective, and motivational processes. 


\section{g. Universal Grammar Theory}

Species Specificity «To say that language is not innate is to say that there is no difference between my granddaughter, a rock and a rabbit. In other words, if you take a rock, a rabbit and my granddaughter and put them in a community where people are talking English, they'll all learn English. If people believe that, then they believe that language is not innate. Clearly, there is something unique about human biological make-up that makes it possible for humans, and only humans, to acquire language. » There are a number of other characteristics which appear to be specific to our species. This suggests there may be a more fundamental difference between humans and the rest of the animal kingdom. Put it, «saying that only humans have language is like saying that only humans build skyscrapers, when the fact is that only humans build freestanding shelters at all». language is a consequence of the basic human ability to recognize others' communicative intentions and to engage in joint attention, which also underlies other cultural achievements. Secondly, it provides the learner with a vital tool for learning language. The point is that arguments for the innateness of language in a general sense do not constitute arguments for the innateness of UG if UG is taken to be a specific body of linguistic knowledge. In other words, the fact that we are the only species that has language does not entail that we have innate knowledge of subjacency. (Chomsky N., 33-54)

\section{Research Methods}

Cognitive theories of human behavior and decision-making models became popular in the early 1970s and 1980s as a response to behaviorism. Cognitive theories explain the mind as the interaction of mental representations and the processes that operate on them. Neither representation nor process is sufficient by itself. Representations do not do anything without processes to operate on them and processes do not do anything without representations to operate on. But put them together and they "execute" like the running of a program. The "steps" they go through are the mind in action, manifest in various behavioral and biological measures, from reaction time and accuracy to event-related electrical brain potentials and functional magnetic resonance imagery. There is an important balance between representation and process. Representations must provide the information that is necessary to support the processing, and the processes must be able to exploit the information available in the representation. Theories that attempt to specify one without the other or focus on one more than the other are incomplete and underdeveloped.

There are two components of cognitivism, cognitive psychology (mental processes) and information processing (cognition as computation). Cognitive psychology is the study of mental processes. These are schema. When presented with new information, learners must: Actively engage with, and organize, information to give it meaning, Practice and rehearse information, Make connections to what is already known and be in an environment that supports learning and take on tasks within their zone of proximal development. Cognitive psychologists study many mental processes, including the following: Attention, Perception, Semantic \& episodic memory, Language development and use, Concepts and categorization, Reasoning and decision making, Problem solving, Procedural and conceptual learning, Consciousness and Skill acquisition.

Schemas are the basic building blocks of such cognitive models, and enable us to form a mental representation of the world. When Piaget talked about the development of a person's mental processes, he was referring to increases in the number and complexity of the schemata that a person had learned. When a child's existing schemas are capable of explaining what it can perceive around it, it is said to be in a state of equilibrium, i. A schema can be defined as 
a set of linked mental representations of the world, which we use both to understand and to respond to situations. The schema is a stored form of the pattern of behavior which includes looking at a menu, ordering food, eating it and paying the bill. This is an example of a type of schema called a 'script. 'Whenever they are in a restaurant, they retrieve this schema from memory and apply it to the situation. The schemas Piaget described tend to be simpler than this - especially those used by infants. He described how - as a child gets older - his or her schemas become more numerous and elaborate. Piaget believed that newborn babies have a small number of innate schemas - even before they have had many opportunities to experience the world. These neonatal schemas are the cognitive structures underlying innate reflexes. Similarly, the grasping reflex which is elicited when something touches the palm of a baby's hand, or the rooting reflex, in which a baby will turn its head towards something which touches its cheek, are innate schemas. Shaking a rattle would be the combination of two schemas, grasping and shaking.

\section{Discussion}

\subsection{Strengths of the Cognitive Theory}

One strength of the cognitive approach is that it has many practical applications. The test could then be used again to help determine if somebody has autism, whilst the knowledge that people with autism or Asperger's syndrome lack theory of mind can help us better understand what autism consists of and how to accommodate this into school or work situations. The cognitive approach heavily relies on experiments as its main research method. Experiments allow for cause and effect to be determined, but more importantly allow for high control over confounding variables.

\subsection{Weaknesses of the Cognitive Theory}

Like all theories, the cognitive perspective is not free from criticism. First, behaviorists see this theory as weak due to the abstract nature of thoughts and the difficulty in defining them. What may be seen as self-critical by one researcher may look like a rational remark by another. Second, there is no agreed upon definition or application of the theory. Because Piaget concentrated on the universal stages of cognitive development and biological maturation, he failed to consider the effect that the social setting and culture may have on cognitive development. The main disadvantage of the cognitive approach is that it refers to cognitive processes that we cannot directly observe. Critics of Loftus and Palmer's leading questions experiments pointed to the validity of the re constructive memory hypothesis, as we cannot be sure that memory has changed as the researchers couldn't observe memories, but only the answers given - which may have been the result of demand characteristics, or even poor judgement of speed. Assuming that findings are the result of invisible processes is heavily subjective and could lead to self-fulfilling prophecy and internal validity being raised as issues. Another weakness of the cognitive approach is that it ignores other factors towards behaviour that have been shown to affect behaviour. For example, Palmer and Hollin's study into moral reasoning and decision making behaviours in criminals assumed that the difference between why the control group weren't criminals and the experimental group were was because of cognitive decision making differences. Some believe Piaget has neglected cultural, educational, and social effect on the cognitive development of children.Piaget did not evaluate children from diverse backgrounds to identify cognitive development stages among children Societal backgrounds; in Western society, he examined only children who had formal education compared to Society outside the West (Lilienfeld, Lynn, Namy, \& Woolf, 2011). 
Others believe that Piaget paid too little attention to the impact of social factors on the cognitive process, however, he did not ignore social influence entirely (Kail, 2012). Piaget explored some aspects of the role of interaction on children, proposing that communication with others transforms a child's egocentric thinking to "socialized thinking" (Lourenço \&at el., 1996). Piaget also acknowledged that cultural context and social interaction affect cognitive development (Shaffer et al., 2010). However, he did not theorize on how social context affects cognitive development (Shaffer et al., 2010). Psychologists now understand children's thinking and understanding by means of their cultural context (Shaffer et al., 2010). Kitchener (1991) believes that Piaget was not familiar with sociology as a science, therefore, Piaget' sociological thought was limited (Kitchener,1991).

The Russian psychologist Lev Vygotsky theorized that interaction with others has a great influence on cognitive development (Hockenbury et al., 2011). He argued that children have the capacity to accomplish larger amounts of cognitive improvement through social interaction, Piaget failed to acknowledge this influence (King, 2011). Piaget established his cognitive development theory based on children's dealing with physical objects, however, Vygotsky believed that a child's mind develops when they interact with other people's minds (Bernstein et al., 2008). During this interaction, children use language to ask questions, and others respond to them, this process contributes to the development in the cognitive ability of children (Cacioppo et al., 2013).

In terms of the principles of developmental science, Piaget's theory has some problems. Firstly, regarding the selection of participants; Piaget did not select a great variety of participants to provide a reliable result, mostly he only examined his own children (Lilienfeld et al., 2011). Furthermore, one of the most important aspects in research methodology is to reduce the effect of sampling bias by selecting participants very carefully. Hence, it is not possible to generalize his ideas to children from different cultures or countries around the world. Secondly, Piaget often utilized the clinical method to collect data. This method is more flexible, so participants are likely to ask different questions (Miller, 2011). However, uniform materials, questions, directions, and techniques to evaluate psychological variables are the spine of experimental psychology (Miller, 2011).

Modern psychologists have been frustrated by Piaget's reports of his experiment. Piaget did not explain the social-economic background of the children, the number of participants, the participant's race or ethnicity, and he did not provide ample detail about his testing measures (Miller, 2011). It is difficult to identify whether Piaget is describing children hypothetically or if he indeed really tested them (Lilienfeld et al., 2011). Moreover, Piaget was poor in the analysis of statistics which he says, "Psychologists over-generalized their methods and arrived at delightful trivialities, particularly when an army of scientists translated their results into mathematical terms" (1918, p. 63 cited in Miller, 2011, 85-86).

In conclusion, there are some criticisms of Piaget's stages. In particular, researchers in the 1960s and 1970s argued that Piaget may have underestimated children's abilities by using confusing terms and particularly difficult tasks in his observations. Other researchers uncovered that there is a range of abilities with cognitive tasks.

In other words, some children may excel or struggle in one area over another. Children may be more adaptable and competent than Piaget's stages give them credit for. Last, Piaget primarily examined white, middle-class children from developed countries in his work. 


\section{a. Critical Evaluation}

Vygotsky and Bruner would rather not talk about stages at all, preferring to see development as a continuous process. Because Piaget concentrated on the universal stages of cognitive development and biological maturation, he failed to consider the effect that the social setting and culture may have on cognitive development. He found that the ability to conserve came later in the aboriginal children, between aged 10 and 13 . However, he found that spatial awareness abilities developed earlier amongst the Aboriginal children than the Swiss children.

Such a study demonstrates cognitive development is not purely dependent on maturation but on cultural factors too - spatial awareness is crucial for nomadic groups of people. Vygotsky, a contemporary of Piaget, argued that social interaction is crucial for cognitive development. This social interaction provides language opportunities and Vygotsky considered language the foundation of thought. Piaget's methods are more open to biased interpretation than other methods. Piaget made careful, detailed naturalistic observations of children, and from these he wrote diary descriptions charting their development. He also used clinical interviews and observations of older children who were able to understand questions and hold conversations. Because Piaget conducted the observations alone the data collected are based on his own subjective interpretation of events. It would have been more reliable if Piaget conducted the observations with another researcher and compared the results afterward to check if they are similar. Such methods meant that Piaget may have formed inaccurate conclusions. As several studies have shown Piaget underestimated the abilities of children because his tests were sometimes confusing or difficult to understand. Piaget failed to distinguish between competence and performance. When Piaget hid objects from babies he found that it wasn't till after nine months that they looked for it. However, Piaget relied on manual search methods whether the child was looking for the object or not. Not only was his sample very small, but it was composed solely of European children from families of high socio-economic status. , thought precedes language. The Russian psychologist Lev Vygotsky argues that the development of language and thought go together and that the origin of reasoning is more to do with our ability to communicate with others than with our interaction with the material world. The influence of Piaget's ideas in developmental psychology has been enormous. He changed how people viewed the child's world and their methods of studying children. He was an inspiration to many who came after and took up his ideas. Piaget's ideas have generated a huge amount of research which has increased our understanding of cognitive development. His ideas have been of practical use in understanding and communicating with children, particularly in the field of education.

\section{b. The Three levels of Adequacy of Cognitive Theory}

Observational adequacy: Development focus on the leaner's capabilities and the learning focuses on the realization of such capabilities and the education within the theory is extrinsic. The cognitive theory, the behavior reflects the emergence of various psychological structures, organized units or patterns of thinking that influence on how children interpret the information.

Descriptive adequacy: .It is absolutely possible to incorporate Piaget's theory in the classroom. Piaget takes a constructivist point of view and believes that learners are not passive in their knowledge. Piaget's theory suggests that students need a curriculum that supports their cognitive development by learning concepts and logical steps.

Explanatory adequacy: The cognitive conflicts occur when the learner is confronted with the information which he/she cannot deal with it in terms of the current cognitive structures. 


\section{Conclusion}

In conclusion, Swiss psychologist Jean Piaget spent his career determining the cognitive ability among children in their early life until a later age. He proposed four cognitive stages, which he believed every child goes through consecutively, without skipping a phase. Although Paget's' theory has had a great influence on the child and developmental psychology, nativism had a different viewpoint in some respects. In terms of the first stage of cognitive development, nativism believed that children are born with some innate knowledge, and they have more capability than Piaget suggested. For example, children can do object permanence, understand the conservation, and imitate others earlier than Piaget proposed. Social factors have had a significant role in cognitive development among children. Children live with their own parents, and they interact with their peers and teachers; all of these have a great deal of influence on a child's level of thinking and understanding. Therefore, Piaget's works have been misinterpreted and criticized in the wrong way, and Piaget had a great deal influence on developmental psychology. Beilin proclaimed that the influence of Piaget's work on developmental psychology is like that of Shakespeare's influence on English literature. Today, the impact of Piaget's theory has guided psychologists in order to reconceptualize the concept of cognitive development. In addition, further researchers have been guided to some important questions which Piaget posed in order to investigate new findings in the area of cognitive development.

\section{References}

Alahmad, Mana. The Role of Form-Focused Instruction on Foreign Language Learners. Budapest International Research and Critics in Linguistics and Education (BirLE) Journal Volume 2, No 4, November 2019, Page: 44-53 e-ISSN: 2655-1470 (Online), pISSN: 2655-2647 (Print) www.bircu-journal.com/index.php/birle

Backscheider, A. G., Shatz, M., \& Gelman, S. a. (1993). Preschoolers' Ability to Distinguish Living Kinds as a Function of Regrowth. Child Development, 64, 1242-1257. https://doi.org/10.2307/1131337

Baillargeon, R. (1987). Object permanence in $31 / 2$ - and 4 1/2-month-old infants. Developmental Psychology,23, 655-664. https://doi.org/10.1037//0012-1649.23.5.655

Bauer, P. J., Larkina, M., \& Deocampo, J. (2011). Early Memory Development. In U. Goswami (Ed.), The Wiley-Blackwell Handbook of Childhood Cognitive Development, Second edition (2nd ed.). New York:Wiley-Blackwell.

Beilin, H. (1992). Piaget's enduring contribution to developmental psychology. Developmental Psychology,28(2), 191-204. https://doi.org/10.1037/00121649.28.2.191

Chomsky N. (1999). "On the nature, use, and acquisition of language," in Handbook of Child Language Acquisition, eds Ritchie W. C., Bhatia T. K. (San Diego, CA: Academic Press; ), 33-54.

Collie, R., \& Hayne, H. (1999). Deferred imitation by 6- and 9-month-old infants: More evidence for declarativememory. Developmental Psychobiology, 35, 83-90. https://doi.org/10.1002/(SICI)1098-2302(199909)35:2<83::AID-DEV1>3.0.CO;2-S

Comer, R., \& Gould, E. (2011). Psychology Around Us. USA: John Wiley \& Sons, Inc.

Doherty, M. J. (2008). Theory of mind: How children understand others' thoughts and feelings. New York:Psychology press. 
Feldman, R. t S. (2013). Essentials of Understanding Psychology. USA: McGraw-Hill.

Gelman, R., \& Baillargeon, R. (1983). A review of some Piagetian concepts. In P. H. Mussen (Ed.), Handbookof Child Psychology. New York: John Wiley \& Sons' Inc.

Piaget, J. (1962). Play, dreams, and imitation in childhood. Morton Library. New York, NY, US: W W Norton\& Co.

Piaget, J., (1964). Part I: Cognitive development in children: Piaget development and learning. Journal of Research in Science Teaching, 2, 3, 176-186.

Rahimirad, Mostafa. The Impact of EFL Teachers' Assessment Literacy on Their Assessment Efficiency in Classroom. Britain International of Linguistics, Arts and Education (BIoLAE) Journal ISSN: 2685-4813 (Online), 2685-4805 (Print) Vol. 1 , No. 1, July 2019, Page: $9-17$

Rogoff, B. (1995). Observing sociocultural activity on three planes: participatory appropriation, guided participation, and apprenticeship. In J. V. Wertsch, P. del Rio, \& A. Alvarez (Eds.), Social Cultural Studies in Mind. Cambridge University Press.

Santrock, J. W. (2011). Child development. New York: McGraw-Hill.

Schacter, D., Gilbert, D., \& Wegner, D. (2011). Psychology. United States of America: Worth Publishers.

Shaffer, D. R., \& Kipp, K. (2010). Developmental Psychology: Childhood and Adolescence. USA: Wadsworth.

Shaughnessy, J. J., Zechmeister, E. B., \& Zechmeister, J. S. (2012). Research Methods in Psychology. USA:McGraw-Hill.

Sigelman, C. K., \& Rider, E. A. (2012). Life-Span Human development. Belmont, USA: Wadsworth, Cengage Learning.

Vygotsky, L.S., (1978). Interaction between learning and development (M. Lopez-Morillas, Trans.). In Cole, M., Steiner, V. J., Scribner, S. \& Souberman, E. Eds., Minds in society: The development of higher psychological processes. Cambridge, MA: Harvard University Press 